\title{
Galaxy transmutations: The double ringed galaxy ESO 474-G26 ${ }^{\star}$
}

\author{
V. Reshetnikov ${ }^{1,2,5}$, F. Bournaud ${ }^{2}$, F. Combes ${ }^{2}$, M. Faúndez-Abans ${ }^{3}$, M. de Oliveira-Abans ${ }^{3}$, \\ W. van Driel ${ }^{4}$, and S. E. Schneider ${ }^{6}$
}

1 Astronomical Institute of St. Petersburg State University, 198504 St. Petersburg, Russia e-mail: resh@astro.spbu.ru

2 Observatoire de Paris, LERMA, 61 Av. de l'Observatoire, 75014 Paris, France

e-mail: frederic. bournaud@obspm.fr

3 MCT/Laboratório Nacional de Astrofísica, Caixa Postal 21, CEP:37.504-364, Itajubá, MG, Brasil

4 Observatoire de Paris, Section de Meudon, GEPI, 5 pl. Jules Janssen, 92195 Meudon, France

5 Isaac Newton Institute of Chile, St. Petersburg Branch, Russia

${ }^{6}$ University of Massachusetts, Astronomy Program, 536 LGRC, Amherst, MA 01003, USA

Received 29 July 2004 / Accepted 22 November 2004

\begin{abstract}
Surface photometry and a $21 \mathrm{~cm} \mathrm{H}$ I line spectrum of the giant double-ringed galaxy ESO 474-G26 are presented. The morphology of this system is unique among the 30000 galaxies with $B \leq 15$. 5. Two almost orthogonal optical rings with diameters of 60 and $40 \mathrm{kpc}$ surround the central body (assuming $H_{0}=70 \mathrm{~km} \mathrm{~s}^{-1} \mathrm{Mpc}^{-1}$ ). The outer one is an equatorial ring, while the inner ring lies in a nearly polar plane. The rings have blue optical colors typical of late-type spirals. Both appear to be rotating around the central galaxy, so that this system can be considered as a kinematically confirmed polar ring galaxy. Its observational characteristics are typical of galaxy merger remnants. Although the central object has a surface brightness distribution typical of elliptical galaxies, it has a higher surface brightness for its effective radius than ordinary ellipticals. Possible origins of this galaxy are discussed and numerical simulations are presented that illustrate the formation of the two rings in the merging process of two spiral galaxies, in which the observed appearance of ESO 474-G26 appears to be a transient stage.
\end{abstract}

Key words. galaxies: individual: ESO 474-G26 - galaxies: photometry - galaxies: formation - galaxies: structure

\section{Introduction}

Polar ring galaxies (PRGs) and related objects are peculiar systems that help to understand the formation of galaxies in general, since they represent extreme cases, providing clues on formation scenarios. The clearest cases of polar rings, named "kinematically confirmed" objects in the Polar Ring Catalog (PRC) of Whitmore et al. (1990), are stable objects frozen in a peculiar morphology, with matter rotating in two nearly perpendicular planes. Numerical simulations have shown that this kind of system could be explained either by simple gas accretion (Reshetnikov \& Sotnikova 1997; and Bournaud \& Combes 2003 , hereafter BC03), or by mergers of perpendicularly oriented disk galaxies (Bekki 1997 \& 1998; BC03). However, in the PRC most objects do not show such a simple appearance with an edge-on central object and an edge-on polar ring, but rather more complex morphologies, in which they are frozen in a quasi-equilibrium state, avoiding complete relaxation

* Based on observations made at the Observatório do Pico dos Dias (OPD), operated by the MCT/Laboratório Nacional de Astrofísica, Brazil. towards a fully mixed symmetric system. These systems with complex morphologies, called polar ring related objects, provide a unique chance to study PRG formation scenarios in objects in which the initial components have not yet disappeared - if they are the results of mergers, it may still be possible to recognize the progenitors in the unrelaxed remnants, and reconstruct their formation events. This is no longer possible in more relaxed merger remnants, that have become elliptical galaxies with only faint shells and ripples.

In this article we present the results of photometric observations and an $\mathrm{HI}$ line spectrum of ESO 474-G26 (PRC C-3 in Whitmore et al. 1990; and AM 0044-243 in Arp \& Madore 1987), a unique galaxy with two almost orthogonal large-scale optical rings (Fig. 1). ESO 474-G26 is in the list of most luminous galaxies (Cappi et al. 1998), and a strong source of far-infrared $\left(\mathrm{NED}^{1}\right)$ and $\mathrm{CO}$ emission (Galletta et al. 1997). Its nuclear spectrum is of an intermediate type - LINER/HII (Sekiguchi \& Wolstencroft 1993). The PRC shows optical emission-line rotation curves along three position angles.

\footnotetext{
${ }^{1}$ NASA/IPAC Extragalactic Database.
} 


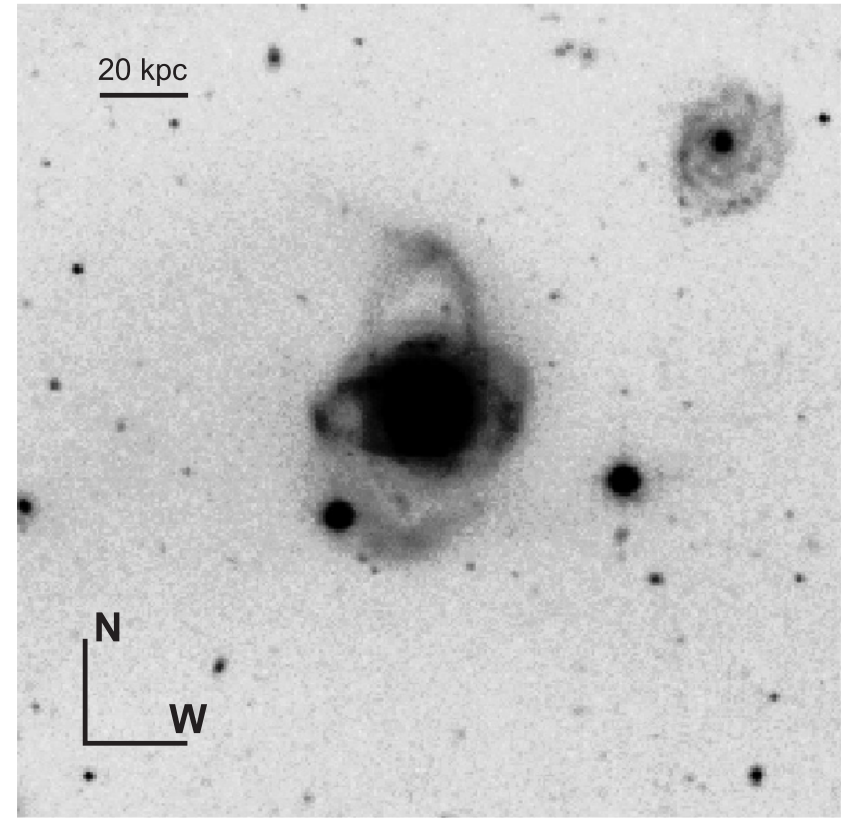

Fig. 1. $V$-band image of ESO 474-G26. The image size is $2 ! 8 \times 2 ! 8$. The spiral to the northwest is a background galaxy.

Observations are detailed in Sect. 2, and results in Sect. 3. A numerical simulation is presented in Sect. 4 to illustrate a possible formation mechanism.

\section{Observations and data reduction}

\subsection{Photometric observations}

The photometric observations were obtained in August, 2002, on the 1.6-m telescope of the Observatório do Pico dos Dias (operated by the MCT/Laboratório Nacional de Astrofísica, Brazil), equipped with direct imaging camera \#1 and a thick, back-illuminated $2048 \times 2048,13.5-\mu \mathrm{m}$ square pixels CCD detector \#98, and scale $0.18 /$ pixel. The readout noise was $2.4 \mathrm{e}^{-}$ and the gain, $2.5 \mathrm{e}^{-} / \mathrm{ADU}$.

The data were acquired with standard Johnson $B, V$ and Cousins $R, I$ band filters. Photometric calibration was made using standard stars from the Landolt (1983) and Graham (1982) lists. The seeing during the observations was 1".3. A log of observations is given in Table 1 , where $Z$ is the zenith angle and $S$ kymag the extinction corrected sky brightness (in mag $\operatorname{arcsec}^{-2}$ ). Reduction of the CCD data was performed in the standard manner using the ESO-MIDAS ${ }^{2}$ package. The $I$ band frames were obtained with exposure times insufficient for detailed photometry, and we used these frames for integral photometry only.

\subsection{HI observations}

The galaxy was observed in the $21 \mathrm{~cm} \mathrm{HI}$ line at the 100 m-class Nançay decimetric radio telescope on 23, 25 and 28 August 2004, for a total of 1 hour per day. For further details

\footnotetext{
${ }^{2}$ MIDAS is developed and maintained by the European Southern Observatory.
}

Table 1. Characteristics of the optical observations.

\begin{tabular}{ccccc}
\hline \hline Dates & $\begin{array}{c}\text { Band- } \\
\text { pass }\end{array}$ & $\begin{array}{c}\text { Exp } \\
(\mathrm{s})\end{array}$ & $\begin{array}{c}Z \\
\left({ }^{\circ}\right)\end{array}$ & $\begin{array}{c}\text { Sky } \\
\mathrm{mag}\end{array}$ \\
\hline 13/14 Aug. 2002 & $B$ & $3 \times 600$ & 4 & 21.9 \\
& $V$ & $3 \times 600$ & 14 & 21.3 \\
& $R$ & $3 \times 600$ & 23 & 20.7 \\
& $I$ & $2 \times 600$ & 6 & 20.1 \\
\hline
\end{tabular}

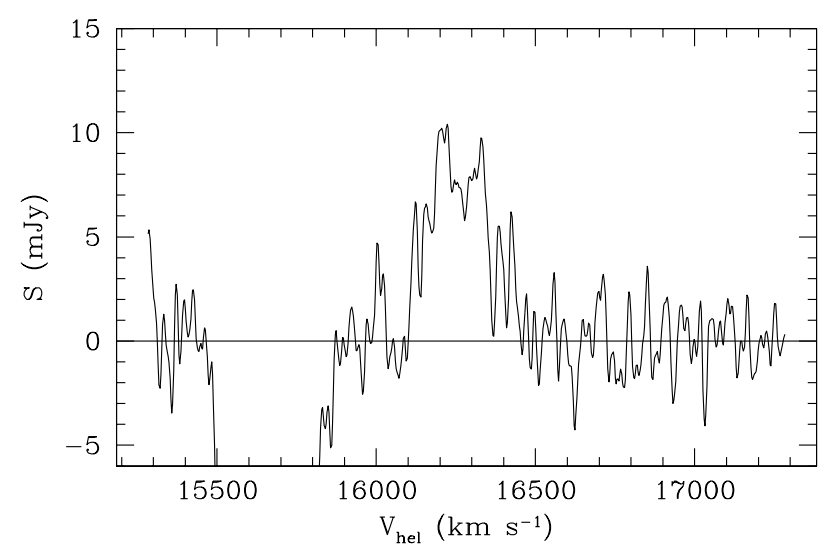

Fig. 2. $21 \mathrm{~cm} \mathrm{H}$ I line spectrum of ESO 474-G26. The negative dip in the $15500-15800 \mathrm{~km} \mathrm{~s}^{-1}$ range is due to imperfectly removed radio frequency interference.

on the telescope, data acquisition and data reduction methods, including the off-line elimination of radio frequency interference (RFI), see Monnier Ragaigne et al. (2001). The half-power beam width of the telescope, of $3.5 \times 23^{\prime}(\alpha \times \delta)$, is expected to cover the entire H I distribution of the galaxy.

In each daily observation a sufficiently wide velocity range around the galaxy profile was found to be free of RFI, in which the galaxy's H I profile was detected in both polarizations. Residual RFI causes the negative dip in the $15500-15800 \mathrm{~km} \mathrm{~s}^{-1}$ range in the averaged data (Fig. 2).

The average of the three spectra (see Fig. 2), smoothed to a velocity resolution of $18 \mathrm{~km} \mathrm{~s}^{-1}$, has an rms noise level of $1.6 \mathrm{mJy}$. The galaxy profile has a peak flux density of $10 \mathrm{mJy}$, a center velocity $V_{\mathrm{HI}}=16249 \pm 15 \mathrm{~km} \mathrm{~s}^{-1}$, a velocity width at $50 \%$ of peak maximum $W_{50}=206 \pm 30 \mathrm{~km} \mathrm{~s}^{-1}$, a velocity width at $20 \%$ of peak maximum $W_{20}=254 \pm 46 \mathrm{~km} \mathrm{~s}^{-1}$, and an integrated line flux $I_{\mathrm{HI}}=1.8 \pm 0.2 \mathrm{Jy} \mathrm{km} \mathrm{s}^{-1}$. These global $\mathrm{HI}$ line parameters are directly measured values; no corrections have been applied to them for, e.g., instrumental resolution. We estimated the uncertainties in $I_{\mathrm{HI}}, V_{\mathrm{HI}}$ and the line widths following Schneider et al. (1986, 1990).

A comparison with the summed $\mathrm{CO}(1-0)$ spectra of Galletta et al. (1997) shows that the widths of the profiles are comparable. The $470 \mathrm{~km} \mathrm{~s}^{-1}$ difference with the published central velocity of the $\mathrm{CO}$ profile could be to due to the application of the relativistic Doppler shift formula to the CO data velocity, without the authors being aware of this correction - converting to the conventional optical definition we use $\left(V=c\left(\lambda-\lambda_{0}\right) / \lambda_{0}\right)$ raises the $\mathrm{CO}$ center velocity to $16233 \pm 11 \mathrm{~km} \mathrm{~s}^{-1}$, consistent with our H I value and the published optical radial velocities. 
Table 2. General properties of ESO 474-G26.

\begin{tabular}{|c|c|c|}
\hline Parameter & Value & Ref. \\
\hline Morphological type & Sb: (merger) & \\
\hline Heliocentric systemic velocity & $16316 \mathrm{~km} \mathrm{~s}^{-1}$ & {$[1]$} \\
\hline Redshift, $z_{3 K}$ & 0.05346 & {$[2]$} \\
\hline $\begin{array}{l}\text { Adopted luminosity distance } \\
\left(\Omega_{m}=0.3, \Omega_{\Lambda}=0.7, h=0.7\right)\end{array}$ & $238 \mathrm{Mpc}$ & \\
\hline Scale & $1^{\prime \prime}=1.0 \mathrm{kpc}$ & \\
\hline Major axis, $\mathrm{D}_{26}\left(\mu_{B}=26\right)$ & $72^{\prime \prime}(72 \mathrm{kpc})$ & \\
\hline Axial ratio, $b / a\left(\mu_{B}=26\right)$ & 0.65 & \\
\hline \multicolumn{3}{|l|}{$\begin{array}{l}\text { Total apparent } \\
\text { magnitudes and colors: }\end{array}$} \\
\hline$V_{\mathrm{T}}$ & $13.98 \pm 0.06$ & \\
\hline$(B-V)_{\mathrm{T}}$ & $+0.75 \pm 0.04$ & \\
\hline$(V-R)_{\mathrm{T}}$ & $+0.49 \pm 0.04$ & \\
\hline$(R-I)_{\mathrm{T}}$ & $+0.44 \pm 0.2$ & \\
\hline$(J-H)_{2 \text { MASS }}$ & +0.67 & [3] \\
\hline$(H-K)_{2 \mathrm{MASS}}$ & +0.35 & {$[3]$} \\
\hline Galactic absorption ( $B$-band) & 0.085 & {$[4]$} \\
\hline $\mathrm{K}$-correction $(B$-band $)$ & 0.122 & [5] \\
\hline Absolute magnitude, $M_{B}^{0}$ & -22.3 & \\
\hline \multicolumn{3}{|l|}{ Central galaxy: } \\
\hline Effective surface brightness, $\mu_{\mathrm{e}}^{0}(B)$ & 21.5 & \\
\hline Effective radius, $R_{\mathrm{e}}$ & $4.8 \mathrm{kpc}$ & \\
\hline Absolute magnitude, $M_{B}^{0}$ & -21.8 & \\
\hline $\mathrm{V}_{\max }^{0}\left(\mathrm{~km} \mathrm{~s}^{-1}\right)$ & 175: & \\
\hline $\begin{array}{l}\text { Central galaxy-to-rings ratio } \\
(B \text {-band) }\end{array}$ & 2: & \\
\hline$M(\mathrm{HI})\left(M_{\odot}\right)$ & $2.2 \times 10^{10}$ & \\
\hline$M\left(\mathrm{H}_{2}\right)\left(M_{\odot}\right)$ & $2.3 \times 10^{10}$ & [6] \\
\hline$L_{F I R}\left(L_{\odot}\right)$ & $1.6 \times 10^{11}$ & {$[2],[7]$} \\
\hline$S F R_{\mathrm{FIR}}\left(M_{\odot} / \mathrm{yr}\right)$ & 43 & \\
\hline$L_{\mathrm{FIR}} / L_{B}$ & 1.1 & \\
\hline$L_{\mathrm{FIR}} / M\left(\mathrm{H}_{2}\right)\left(L_{\odot} / M_{\odot}\right)$ & 7 & \\
\hline$M\left(\mathrm{H}_{2}\right) / L_{B}\left(M_{\odot} / L_{\odot, B}\right)$ & 0.18 & \\
\hline$M(\mathrm{HI}) / L_{B}\left(M_{\odot} / L_{\odot, B}\right)$ & 0.17 & \\
\hline
\end{tabular}

[1] - HyperLEDA mean value; [2] - NASA/IPAC Extragalactic Database (NED); [3] - Skrutskie et al. (1997); [4] - Schlegel et al. (1998); [5] - Bicker et al. (2004); [6] - Galletta et al. (1997); [7] Based on the IRAS 60 and $100 \mu \mathrm{m}$ flux densities

\section{Results}

\subsection{General structure}

ESO 474-G26 is a large system - even the central galaxy (without the rings) is significantly larger and much more luminous than the Milky Way (Table 2).

The $V$-band image is displayed in Fig. 1 and represented as an isophotal plot in Fig. 3. The overall optical morphology of ESO 474-G26 is very interesting and intriguing: a nearly spherical central body is surrounded by two almost perpendicular giant rings. Both ring-like structures look somewhat irregular.

The galaxy colors (after Galactic reddening correction and $\mathrm{K}$-correction for an early-type galaxy) correspond to those of an Sb-type spiral. Figure 4 presents the spectral energy

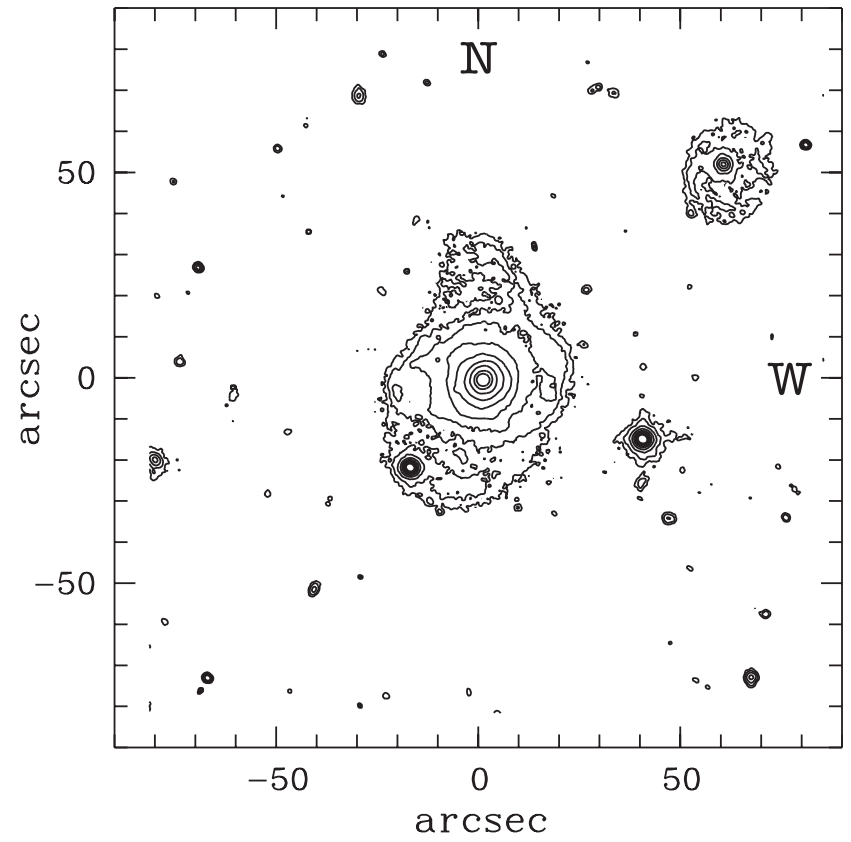

Fig. 3. Isophotal contour map of ESO 474-G26 in the $V$ band. The faintest contour is $25.8 \mathrm{mag} \operatorname{arcsec}^{-2}$, and the isophote step - 0.75 .

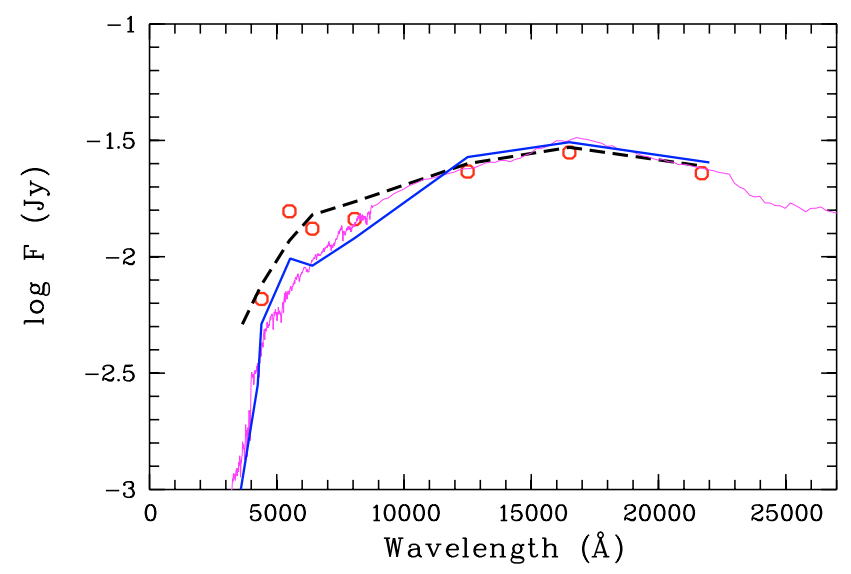

Fig. 4. Spectral energy distribution for ESO 474-G26 (circles). The thick solid line represents the distribution for the giant $\mathrm{Sab} / \mathrm{Sb}$ galaxy M 81 according to the NED data, the thin solid line the SED for an Sb galaxy model (Fioc \& Rocca-Volmerange 1997), and the dashed line the SED for the merger NGC 7252 (NED). All the SEDs have been arbitrarily shifted to fit approximately the observed SED of ESO 474-G26. BVRI data are from our photometry.

distribution (SED) for ESO 474-G26. As can be seen, although both observed and modeled SED curves for Sb galaxies fit well the observed SED from the $I$ band to larger wavelengths, the SED for the prototype advanced merger remnant "Atoms-forPeace" galaxy, NGC 7252 (e.g., Schweizer 1982) gives a much better approximation (see further discussion).

ESO 474-G26 is a strong source of far-infrared emission (Table 2). The detected far-infrared luminosity, converted to a star formation rate, indicates a very high rate of star formation (Table 2), as does the large $L_{\mathrm{FIR}} / L_{B}$ ratio of 1.1. The ratio of the $\mathrm{H}_{2}$ mass to blue luminosity $\left(0.18 M_{\odot} / L_{\odot, B}\right)$ is typical of Sb-Sbc 

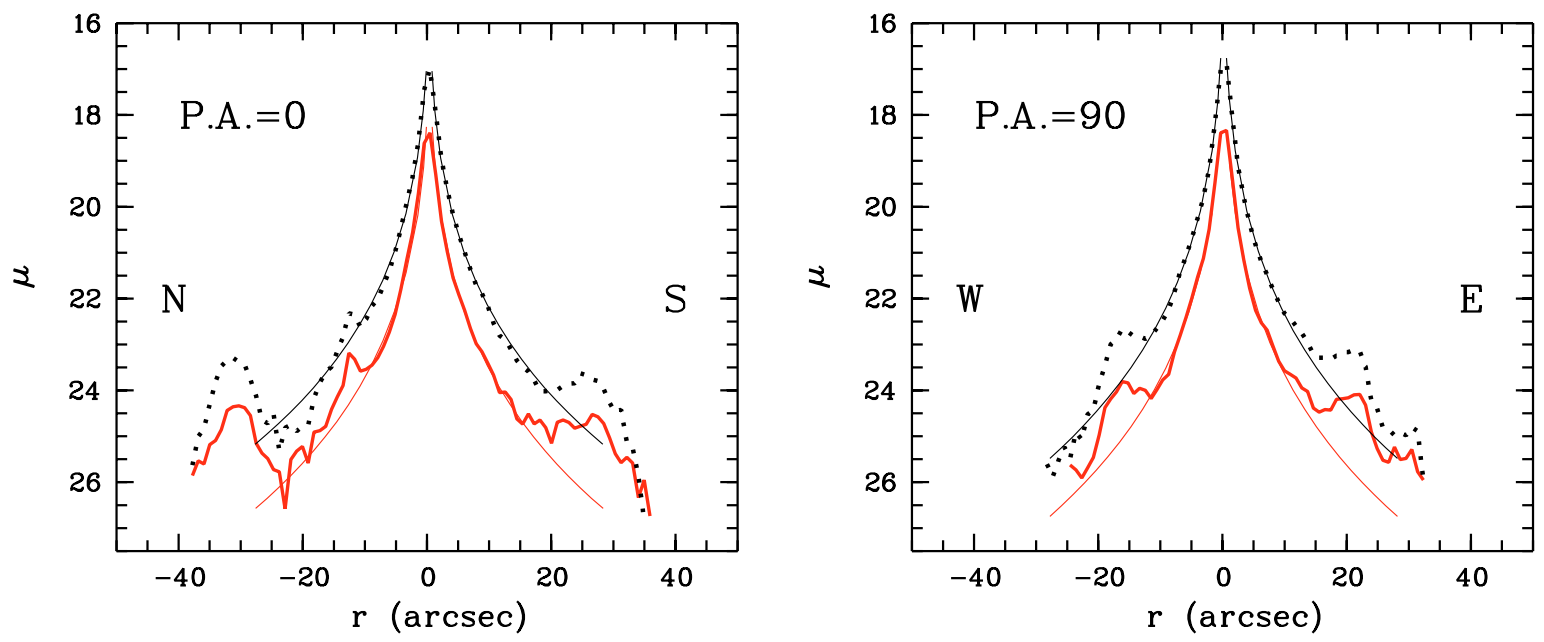

Fig. 5. Photometric profiles for ESO 474-G26 at two position angles. Thick solid lines represent the distributions in the $B$ passband, and dotted ones those in the $R$. Thin lines show fits to the profiles by the de Vaucouleurs' law.
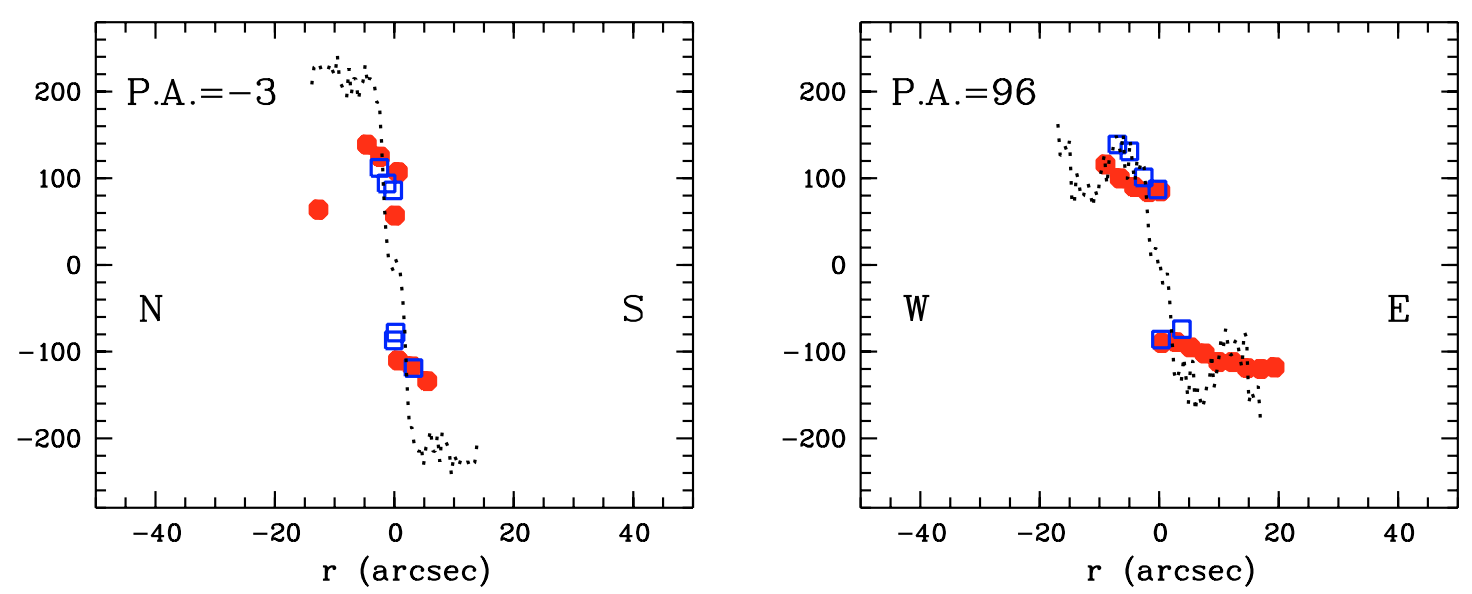

Fig. 6. Emission-line radial velocities (in $\mathrm{km} \mathrm{s}^{-1}$ ) along two position angles, according to the PRC. The filled circles are from H $\alpha$ measurements, and the squares are from [NII]6583 Å measurements. Dotted lines represent the corresponding radial velocity distributions for the model galaxy (see Sect. 4). Observed velocities are from Whitmore et al. (1990), and are relative to heliocentric velocity of $16268 \mathrm{~km} \mathrm{~s}^{-1}$.

spirals (Young \& Knezek 1989), as are the relative H I content and the $\mathrm{H}_{2}$ to $\mathrm{H}$ I ratios (Table 2).

A faint background galaxy (with a radial velocity of about $32000 \mathrm{~km} \mathrm{~s}^{-1}$ - see the PRC) is located $80^{\prime \prime}$ NW of ESO 474G26 (Figs. 1, 3). The total apparent magnitude of the galaxy is $V_{\mathrm{T}}=17.1 \pm 0.1$ and its colors are $(B-V)_{\mathrm{T}}=+0.82,(V-R)_{\mathrm{T}}=$ $+0.30,(R-I)_{\mathrm{T}} \approx 0.0$. Therefore, this is a giant (with an absolute magnitude in the $B$ band of about -20.7) late-type (from optical morphology and colors) spiral galaxy.

General properties of ESO 474-G26 are summarized in Table 2.

\subsection{Central galaxy}

The central object has almost round isophotes (Fig. 3) with apparent axial ratios in the $R$ passband of $\langle b / a\rangle=$ $0.94 \pm 0.015$ within $15^{\prime \prime}$ of the center. Photometric profiles at two position angles are shown in Fig. 5. At $|r| \leq 10^{\prime \prime}$ (or within $10 \mathrm{kpc}$ from the nucleus), the surface brightness can be well approximated by the de Vaucouleurs' law. The observed colors of the galaxy within $r=10^{\prime \prime}(B-V=0.80, V-R=0.51)$ are somewhat redder than for the galaxy as a whole (Table 2) but bluer than typical values for ellipticals.

A comparison of the effective parameters $-\mu_{\mathrm{e}}, R_{\mathrm{e}}-$ of the central galaxy with the Kormendy relation (photometric projection of the Fundamental Plane, Kormendy 1977) shows an enhancement in $\mu_{\mathrm{e}}$ of about $1.0-1 \mathrm{~m}$. 5 in $B$ at a fixed $R_{\mathrm{e}}$ (e.g., Capaccioli et al. 1992), or, at a fixed surface brightness, that the galaxy has an effective radius 2-3 times larger than ordinary ellipticals.

Optical spectra showing the gas kinematics in ESO 474-G26 along three different position angles are given in the PRC. We reproduce in Fig. 6 the distributions of radial velocities at two position angles which are relatively close to the major axes of the rings. The central galaxy of ESO 474-G26 is surrounded by two ring-like structure, one polar and one equatorial. Both rings are in rotation around the galaxy center. Therefore, ESO 474-G26 can be formally 


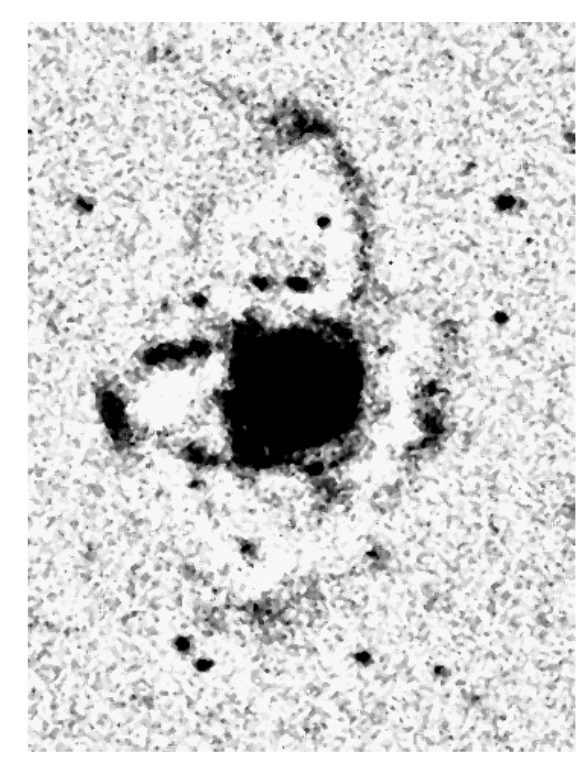

Fig. 7. Residual $R$ band image after the subtraction of the $8^{\prime \prime} .8 \times 8^{\prime \prime} .8$ median filtered frame from the original image. The image size is 1 '.44 $\times 1$ '.08. North is up, and East is left.

classified as a kinematically confirmed PRG. The third spectrum published in the PRC shows the rotation of gas at a position angle of $132^{\circ}$, which is intermediate between the two rings and reveals the complex kinematical structure of ESO 474-G26.

The observed maximum rotation velocity of the galaxy is $V_{\text {max }} \approx 140 \mathrm{~km} \mathrm{~s}^{-1}$ at $r \approx 5^{\prime \prime}$ along PA $=-3^{\circ}$ (PRC). The apparent axial ratio of this ring is $\langle b / a\rangle=0.6 \pm 0.1$. Assuming that the emission lines belong to the large ring, and that this ring is intrinsically circular, we can estimate its inclination as $i=53^{\circ} \pm 7^{\circ}$. Therefore, corrected for the inclination, the maximum rotation velocity at $r=5^{\prime \prime}=5 \mathrm{kpc}$ is $V_{\max }^{0} \approx 175 \mathrm{~km} \mathrm{~s}^{-1}$. The mass to luminosity ratio is $M / L_{B}\left(r \leq 5^{\prime \prime}\right) \approx 1 M_{\odot} / L_{\odot, B}$, a value that is unusual for early-type galaxies and more typical of spirals with active star formation. Due to the highly uncertain geometry of the galaxy, the above estimates of $V_{\max }$ and $M / L_{B}$ are tentative only.

\subsection{Rings}

The major axis position angle of the first (smaller) ring is $94^{\circ}$, and its diameter is $\approx 37^{\prime \prime}$ or $37 \mathrm{kpc}$. The second (larger) ring has $\mathrm{PA}=170^{\circ}$ and diameter of $58^{\prime \prime}(58 \mathrm{kpc})$. In projection, the angle between the two rings is $76^{\circ}$.

The optical colors of the rings were determined through a $10^{\prime \prime}$ diameter circular aperture placed on the brightest parts of the rings along its major axes. In the large ring we found the following colors at the indicated positions with respect to the nucleus:

$B-V=0.69, V-R=0.38\left(32^{\prime \prime} \mathrm{N}\right)$,

$B-V=0.56, V-R=0.36\left(27^{\prime \prime} \mathrm{S}\right)$;

and for the smaller ring:

$B-V=0.69, V-R=0.48\left(17^{\prime \prime} \mathrm{W}\right)$,

$B-V=0.54, V-R=0.43\left(21^{\prime \prime} \mathrm{E}\right)$.

Therefore, both rings are bluer than the central galaxy (see

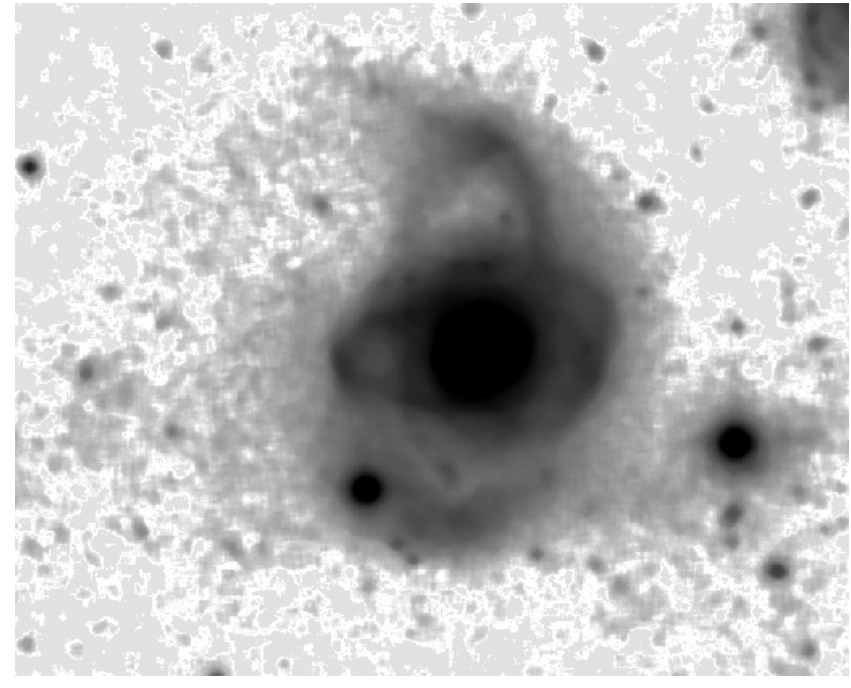

Fig. 8. Deep, high-contrast image of ESO 474-G26. The image size is $1: 8 \times 2 ! 1$

previous section), and its extinction and redshift corrected colors $(B-V \approx 0.5, V-R \approx 0.4)$ are typical for Sc-Scd spirals (Buta et al. 1994; Buta \& Williams 1995) and similar to those of PRG rings (Reshetnikov et al. 1994, 1995).

Both rings show a color asymmetry, in the sense that their $\mathrm{N}$ and $\mathrm{W}$ parts are redder, which is not unusual for polar rings - see for instance the correlation found between the largescale color asymmetry in the rings of the PRGs UGC 7576 and UGC 9796.

Various techniques of image enhancement have been employed to reveal fine structure within ESO 474-G26 (see, for example, Faúndez-Abans \& de Oliveira-Abans 1998, for a description of some techniques). In Fig. 7 we present the residual image of the galaxy. The smaller (east - west) ring looks very irregular, with many condensations. The brightest condensations show $B \approx 21$ (or $M_{B} \approx-16$ ) and angular sizes around $2^{\prime \prime}$ ( $2 \mathrm{kpc})$. Most probably, the condensations represent giant H II regions.

The larger outer (north-south) ring has the southern part approaching (PRC, Galletta et al. 1997). The western part of the ring is probably the nearest to us (from the dust lane crossing the western side of the central body - see Fig. 1). In Fig. 8 we present a deep $(B+V+R)$ and smoothed image of ESO 474-G26. A faint $\left(\mu(V) \geq 26^{\mathrm{m}}\right)$ asymmetric envelope extends to the east. The North and South edges of the large ring show extensions towards the east and west, respectively; they might be spiral arms.

\section{Discussion and conclusions}

The true geometrical structure and the possible origin of ESO 474-G26 are intriguing mysteries. A number of formation scenarios - resonance ring, accretion on a ringed earlytype galaxy, and a minor merger - can be excluded, for the following reasons:

- Although the larger ring reveals some spiral structure, it cannot be an outer pseudo-ring of a resonant nature (e.g., 

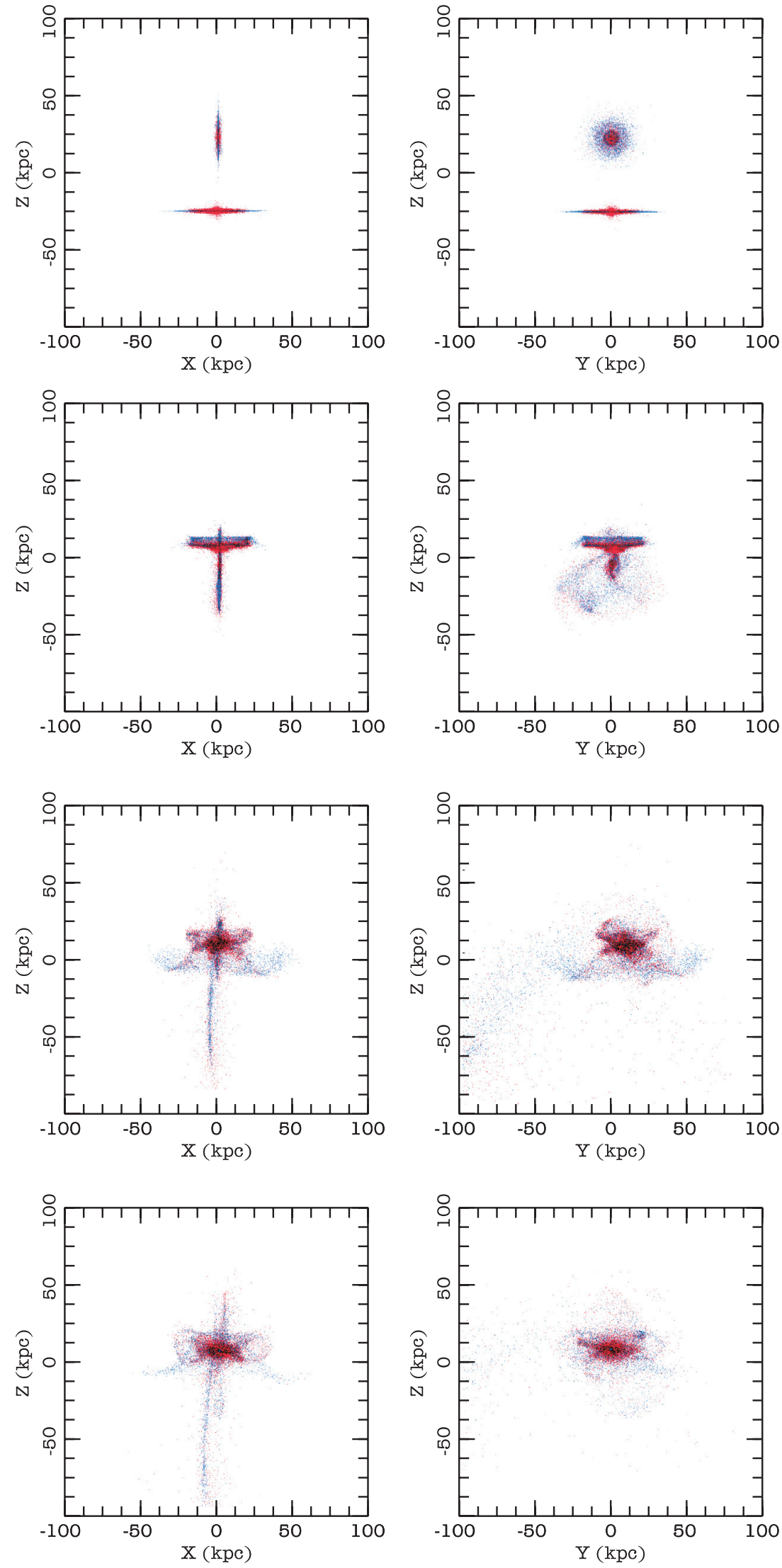

Fig. 9. Two orthogonal projections, $(x, y)$ and $(z, y)$, of the galaxy merging process (one projection per column). Epochs after the beginning of the simulations are $t=200,350,500$, and 650 Myrs (from top to bottom). Red points represent stars, blue points represent gas. 


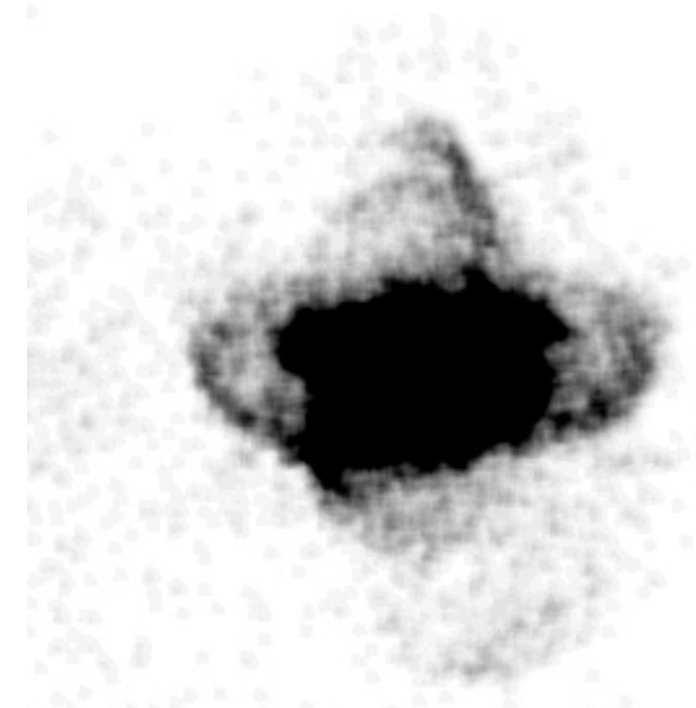

Fig. 10. Projected distribution of stars in the simulated merger at $t=700$ Myrs. The image size is $100 \mathrm{kpc} \times 100 \mathrm{kpc}$. The projection was selected to fit the appearance of ESO 474-G26.

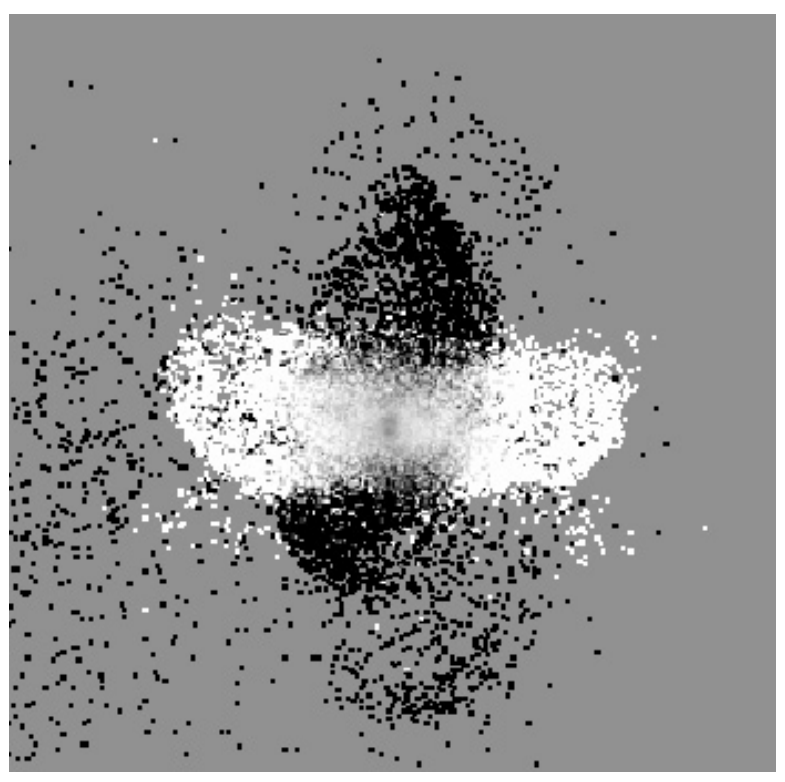

Fig. 11. Ratio map of the stellar populations from the victim and intruder galaxies. White: $100 \%$ of the stars come from the victim disk (victim's stars or stars formed in gas from the victim disk) - black: $100 \%$ of the stars come from the intruder's material - background gray: 50\% from each galaxy, or no stars. The scale is linear. Each ring owes more than $98 \%$ of its stellar mass to one of the pre-existing galaxies, and there is no mixing. In the central $500 \mathrm{pc}$, on the contrary, each galaxy contributes about $50 \%$ to the stellar mass.

Buta \& Combes 1996), since the central galaxy is clearly not the barred disk galaxy required to form such a ring.

- The irregular and unrelaxed aspect of the system could indicate a relatively recent external polar accretion from a gas-rich donor galaxy onto a pre-existing early-type galaxy with an outer ring. If so, one would expect to find the relic of the donor galaxy within a reasonable distance from
ESO 474-G26, but ESO 474-G26 appears to be isolated and without a companion within $1 \mathrm{Mpc}$ projected distance at similar redshift.

- A minor merger with a peculiar geometry that formed the inner polar ring is unlikely to have resulted in the observed unperturbed outer pseudo-ring.

It is appropriate to test a major merger scenario that forms both rings at the same time, since, globally, ESO 474-G26 shows analogies to the prototypical merger remnant NGC 7252 (Schweizer 1982) - which is also a giant galaxy, with an elliptical-like surface brightness profile, relatively blue colors, peculiar kinematics, outer structures, and a similar spectral energy distribution (as shown in Fig. 4).

To reproduce ESO 474-G26 in detail, we have to solve a reverse problem: to find the characteristics and orientations of the progenitor galaxies (as well as their orbits) from the observed structure of the merger remnant. Of course, this is not a fully determined problem, and many pieces of observational data are missing, like the 2D gaseous and stellar kinematics of the system. Therefore, we choose to find a generic solution only that reproduces a double ringed optical appearance with the right relative proportions of the galaxy.

On the basis of the results by Bekki (1998) and BC03, we have considered a low-velocity head-on collision between two galaxies with orthogonal spiral disks. The simulations were performed with an $\mathrm{N}$-body particle code which includes gas dynamics, star formation and stellar mass-loss (see Bournaud \& Combes 2002, 2003, for details).

After several trials, we found an acceptable solution (Figs. 9 and 10). The main galaxy, hereafter denoted as the victim (as in Bekki 1998), is a giant spiral galaxy (the exponential scalelength of the stellar disk is $5.3 \mathrm{kpc}$ ) with a total mass $M_{\text {tot }}=6.1 \times 10^{11} M_{\odot}$, a bulge-to-disk luminosity ratio 0.25 , and a relatively low gas fraction $M_{\text {gas }} / M_{\text {stars }}=0.03$. The galaxy is surrounded by a moderately massive spherical dark halo with a mass ratio $M_{\mathrm{DH}} / M_{\mathrm{stars}}=1.2$. The second galaxy, the intruder, has a total mass of $M_{\text {tot }}=2.7 \times 10^{11} M_{\odot}$, an exponential scalelength of its stellar disk of $2.4 \mathrm{kpc}, M_{\text {gas }} / M_{\text {stars }}=0.065$, and $M_{\mathrm{DH}} / M_{\text {stars }}=1.1$. Initially, the galaxies were separated by $70 \mathrm{kpc}$ and their relative velocity was $70 \mathrm{~km} \mathrm{~s}^{-1}$.

Figure 9 shows the morphological evolution of the galaxies during the merging process. The total number of particles used in this simulation is $2 \times 10^{5}$. At $t=700 \mathrm{Myr}$ (or about $350 \mathrm{Myr}$ after the first crossing), we show in Fig. 10 a projection of the system that is selected to mimic the observed double-ring appearance. At this time, the merger remnant shows a ring-like structure along its apparent minor axis: this ring consists of tidal debris from the intruder, and is polar with respect to the victim disk. At the same time, an expanding collisional ring of the Cartwheel type is formed by the victim galaxy material, which is polar with respect to the intruder galaxy's initial plane. Thus, we find the two types of rings studied in $\mathrm{BC} 03$ around the same object. The diameter of the collisional ring is $60 \mathrm{kpc}$, in agreement with the size of the large ring of ESO 474-G26 (Sect. 3.3). As one can see in Fig. 6, our model fits the observed rotation curve of ESO 474-G26 along the small ring, and is not inconsistent with the major axis kinematics, at least 
in the brightest regions of this ring. We show in Fig. 11 the composition of the two rings: the small, tidal ring is only made up of stars from the intruder galaxy (i.e., stars that initially were in the intruder disk, or stars formed recently from the intruder's gas), and the large ring is only made up of stars from the victim galaxy. The degree of mixing of the stellar populations in the rings is less than $2 \%$.

There are two differences between ESO 474-G26 and our artificial merger. The first is that the ratio of the brightness of the rings to that of the peak in the central galaxy is half the value in the model to that actually observed. However, we have assumed a constant mass-to-light ratio in the model. As the rings mainly contain young stars, they could have a lower $M / L$ ratio than the central galaxy, which solves this problem. The second difference is that the inner part of the model remnant looks unrelaxed in comparison to the observed smooth, elliptical-like surface brightness distribution in ESO 474-G26.

In the simulations, the small ring, or the vertical coherent structure (see Fig. 10) is a transient feature. It persists for about $200 \mathrm{Myr}$ and then disperses and vanishes. Approximately 1 Gyr after the full merging, the remnant becomes smooth and elliptical-like. Therefore, the impressive double-ring appearance of ESO 474-G26 could also be transient and not reflect a stable dynamical configuration.

It appears likely that we are indeed observing a transient stage in a particular merging scenario, since ESO 474-G26 is the only clear example with two such rings among $\approx 3 \times 10^{4}$ known galaxies with $B \leq 15$. 5 (PRC, NED).

We thus conclude that one of the most plausible solutions is that ESO 474-G26 represents a transient phase of a merging process between two spiral galaxies. This is based on its observational characteristics, which are typical of merger remnants, as well as on numerical simulations that reproduce the general morphology of the galaxy.

Acknowledgements. We are grateful to the referee, C. Horellou, for her constructive comments. This work was carried out while VR was visiting the LERMA in Paris, thanks to a CNRS 3-month visitor grant. V.R. acknowledges support from the Russian Foundation for Basic Research (03-02-17152) and from the Russian Federal Program "Astronomy" (40.022.1.1.1101). M.F.-A. and M.deO.-A. acknowledge the partial support of the Fundação de Amparo ã Pesquisa do Estado de Minas Gerais (FAPEMIG) and the Ministério da Ciéncia e Tecnologia (MCT, Brazil). The computations have been carried out on the Fujitsu NEC-SX5 of the CNRS computing center, at IDRIS.
The Nançay Radio Observatory, which is the Unité Scientifique de Nançay of the Observatoire de Paris, is associated with the French Centre National de Recherche Scientifique (CNRS) as USR B704, and acknowledges the financial support of the Région Centre as well as of the European Union.

\section{References}

Arp, H. C., \& Madore, B. F. 1987, in A Catalogue of Southern Peculiar Galaxies and Associations (Cambridge: Cambridge Univ. Press)

Bekki, K. 1997, ApJ, 490, L37

Bekki, K. 1998, ApJ, 499, 635

Bicker, J., Fritze-v.Alvensleben, U., \& Fricke, K. J. 2004, A\&A, 413, 37

Bournaud, F., \& Combes, F. 2002, A\&A, 392, 83

Bournaud, F., \& Combes, F. 2003, A\&A, 401, 817 (BC03)

Buta, R., Mitra, S., de Vaucouleurs, G., \& Corwin, H. G. 1994, AJ, 107,118

Buta, R., \& Williams, K. L. 1995, AJ, 109, 543

Buta, R., \& Combes, F. 1996, Fund. Cosm. Phys., 17, 95

Capaccioli, M., Caon, M., \& D'Onofrio, M. 1992, MNRAS, 259, 323

Cappi, A., da Costa, L. N., Benoist, C., et al. 1998, AJ, 115, 2250

Faúndez-Abans, M., \& de Oliveira-Abans, M. 1998, A\&AS, 128, 289

Fioc, M., \& Rocca-Volmerange, B. 1997, A\&A, 326, 950

Galletta, G., Sage, L. J., \& Sparke, L. S. 1997, MNRAS, 284, 773

Graham, J. A. 1982, PASP, 94, 244

Landolt, A. U. 1983, AJ, 88, 439

Kormendy, J. 1977, ApJ, 218, 333

Monnier Ragaigne, D., van Driel, W., Schneider, S. E., Balkowski, C., \& Jarrett, T. H. 2003, A\&A, 408, 465

Reshetnikov, V. P., Hagen-Thorn, V. A., \& Yakovleva, V. A. 1994, A\&A, 290, 693

Reshetnikov, V. P., Hagen-Thorn, V. A., \& Yakovleva, V. A. 1995, A\&A, 303, 398

Reshetnikov, V., \& Sotnikova, N. 1997, A\&A, 325, 933

Schlegel, D. J., Finkbeiner, D. P., \& Davis, M. 1998, ApJ, 500, 525

Schneider, S. E., Helou, G., Salpeter, E. E., \& Terzian, Y. 1986, AJ, 92,742

Schneider, S. E., Thuan T. X., Magri, C., \& Wadiak, J. E. 1990, ApJS, 72,245

Schweizer, F. 1982, ApJ, 252, 455

Sekiguchi, K., \& Wolstencroft, R. D. 1993, MNRAS, 263, 349

Skrutskie, M. F., Schneider, S. E., Steining, R., et al. 1997, in The Impact of Large Scale Near-IR Sky Surveys, ed. F. Garzon et al. (Dordrecht: Kluwer), 25

Tully, R. B., Pierce, M. J., Huang, J.-Sh., et al. 1998, AJ, 115, 2264

Whitmore, B. C., Lucas, R. A., McElroy, D. B., et al. 1990, AJ, 100, 1489 (PRC)

Young, J. S., \& Knezek, P. M. 1989, ApJ, 347, L55 